\title{
Imaging recommendations in paediatric uroradiology: minutes of the ESPR workgroup session on urinary tract infection, fetal hydronephrosis, urinary tract ultrasonography and voiding cystourethrography, Barcelona, Spain, June 2007
}

\author{
Michael Riccabona • Fred E. Avni • \\ Johan G. Blickman • Jean-Nicolas Dacher • \\ Kassa Darge • M. Luisa Lobo • Ulrich Willi
}

Received: 1 October 2007 / Accepted: 22 October 2007 / Published online: 11 December 2007

(C) Springer-Verlag 2007

\begin{abstract}
We present here a few basic proposals for algorithms and procedures for imaging the paediatric genitourinary tract based on initial discussion at a paediatric uroradiology symposium and proposals of the ESUR Paediatric Uroradiologic Guidelines Subcommittee. These recommendations were developed in the light of new knowledge that might influence existing guidelines. Regional, individual and local flexibility and variability should be preserved in order to make these recommendations applicable throughout Europe. They should help standardize dedicated imaging not only in terms of a quality measure to ensure state-of-the-art patient care, but also in forming a common basis for multi-institutional research.
\end{abstract}

This work is presented on behalf of the ESUR Paediatric Guideline Subcommittee and ESPR Paediatric Uroradiology Working Group

M. Riccabona $(\square)$

Department of Radiology, Division of Pediatric Radiology,

University Hospital LKH Graz,

Auenbruggerplatz,

A-8036 Graz, Austria

e-mail: michael.riccabona@klinikum-graz.at

e-mail: michael.riccabona@meduni-graz.at

F. E. Avni

Department of Medical Imaging, University Clinics of Brussels, Erasme Hospital,

Brussels, Belgium

J. G. Blickman

Department of Radiology, Radboud University Medical Center,

Nijmegen, The Netherlands

J.-N. Dacher

Department of Radiology, University Hospital of Rouen,

Rouen, France
There is an urgent need for these guidelines in order to advance our understanding of the subject and to gain evidence and improve imaging efficacy. Our session worked towards establishing an agreement on imaging indications in common paediatric urological conditions, respecting the ALARA principle, and patient safety and care, and taking into account state of the art knowledge and efficacy aspects. We started the task with a reassessment of (1) imaging in urinary tract infection in infants and children, (2) postnatal imaging in mildto-moderate neonatal hydronephrosis, (3) how to perform voiding cystourethrography, and (4) procedural recommendations for paediatric urosonography. This list is incomplete, and

\section{K. Darge}

Division of Body Imaging, Department of Radiology,

The Children's Hospital of Philadelphia (CHOP),

University of Pennsylvania,

324 South 34th Street,

Philadelphia, PA 19104, USA

M. L. Lobo

Department of Radiology, Santa Maria Hospital,

University Hospital,

Lisbon, Portugal

U. Willi

Department of Radiology, Lucile Packard Children's Hospital,

Stanford University,

Stanford, CA, USA 
future recommendations will be developed, discussed and presented at forthcoming meetings.

Keywords Uroradiology · Paediatric . Imaging recommendations · Urinary tract infection . Fetal hydronephrosis

\section{Introduction}

Paediatric uroradiology is one of the mainstays of daily paediatric imaging practice. The number of infants and children submitted for assessment of the urinary tract has grown significantly, since fetal US screening has become routine. This has resulted in an increasing number of babies being evaluated for prenatally detected "hydronephrosis" (HN). When studying these children and observing their further course, new knowledge and insight has been gained that sheds new light on the accepted traditional imaging algorithms. In particular, neonatal vesicoureteral reflux (VUR) is now thought to be often a transitory condition that in up to $80 \%$ of patients diminishes or disappears spontaneously, even in those with high-grade VUR. We also learned that VUR itself does not impair renal function after the fetal period. High-grade neonatal VUR (mostly in boys) is often associated with prenatally developed renal dysplasia associated with high-grade HN (Fig. 1), whereas most other forms of primary or congenital VUR are not necessarily associated with renal damage or long-term sequelae. As therapeutic concepts are changing and even the necessity and usefulness of long-term antibiotic prophylaxis is being discussed and new surgical options such as cystoscopic subureteric injection are now available, the impact of imaging results on patient management is changing and indications for various procedures need to be reassessed.

Traditionally, VUR has been considered the major risk factor for urinary tract infection (UTI) and renal damage, with potentially severe long-term sequelae such as hypertension and impaired renal function. It has been shown that UTI and renal scarring occur without VUR and that, even in patients with VUR, UTI does not necessarily cause renal damage and scarring, while about half the patients with renal scarring do not have or never had VUR. On the other hand, we know that the incidence of renal involvement and potential scarring after UTI is significantly higher in those patients with medium-to-high-grade VUR, and that the diagnosis of VUR may be missed. Thus new strategies are being developed, focusing more on preserving renal parenchyma than on VUR detection and management, with increasing interest in bladder function. Several other factors determine the risk of renal damage, such as the time of diagnosis and onset of treatment, the virulence of the bacteria, bacterial resistance to various antibiotics, immunological competence of the urothelium, intravesical pressure, and genetic and some anatomic predispositions (e.g. compound papillae or underlying urinary tract malformation). For these reasons imaging strategies in UTI are changing. They range from exhaustive imaging workup to not performing any imaging at all. We evaluated the various arguments carefully and recommend limiting and possibly avoiding invasive investigations, especially procedures that use ionizing radiation, for economic reasons, as well as to
Fig. 1 Grading of hydronephrosis in neonates and infants (adapted from the fetal "SFU classification" for postnatal use; Fernbach SK, Maizels $\mathrm{M}$, Conway JJ. Ultrasound grading of hydronephrosis: introduction to the system used by the Society for Fetal Urology. Pediatr Radiol 1993; 23:478480)

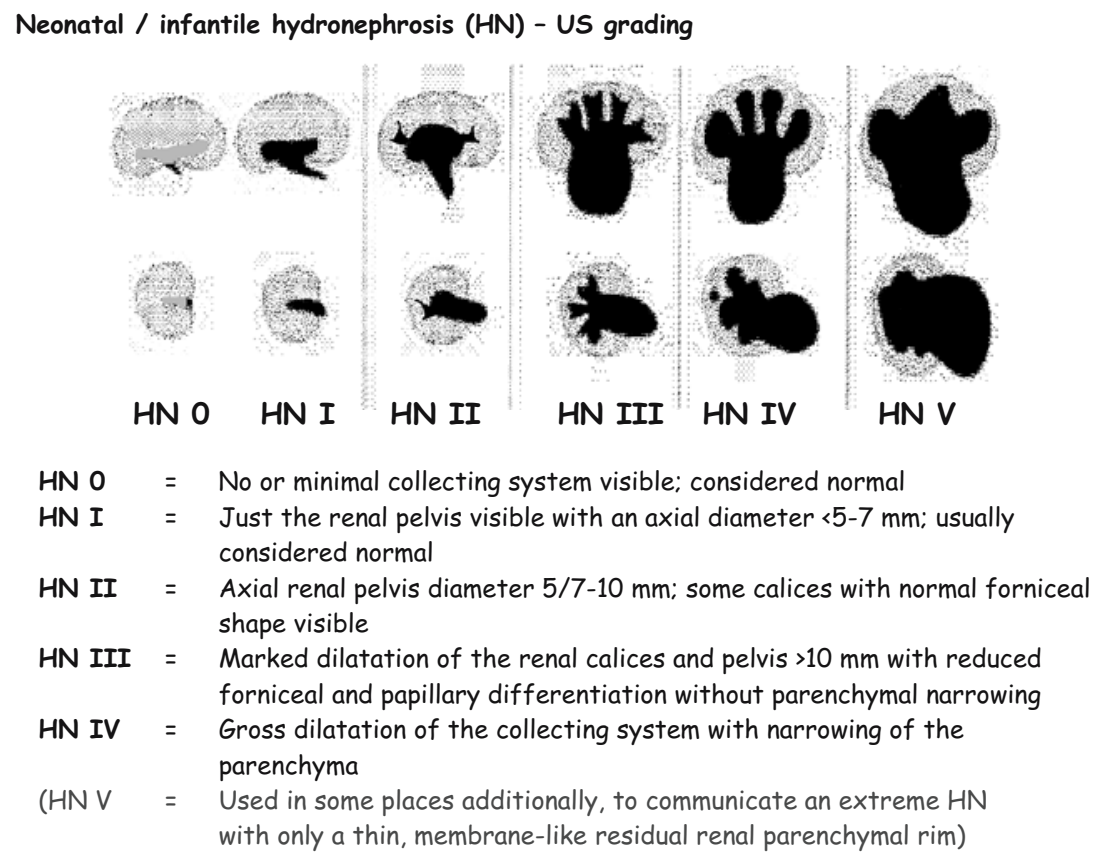


improve patient care. On the other hand, there are benefits deriving from the traditional imaging protocols that should be preserved. Although there is no conclusive evidence that traditional imaging significantly impacts long-term patient outcome, there is also no certainty that these studies can be abandoned without endangering the patients' health and long-term outcome.

Additionally, the technique and scope of imaging procedures have evolved. By using modern X-ray equipment and following the ALARA principle with careful coning and elimination of unnecessary radiation (e.g. grid, large field-of-view, etc voiding cystourethrography; VCUG) may be performed with a large dose reduction. Ultrasonographic resolution and colour Doppler techniques have been markedly improved and new US techniques have been introduced. Ultrasonography has developed from a roughly orienting initial study to a powerful diagnostic tool. Finally, new imaging methods have been introduced and have become commonly used in paediatric imaging, such as US contrast media, magnetic resonance urography (MRU) and multidetector CT (MDCT). All this needs to be considered in relation to imaging algorithms and standard recommendations on how the various imaging modalities should be applied and how the various conditions should be best approached.

Increasing efforts throughout the medical community to standardize diagnostic imaging to ensure proper patient management, increasing acceptance of quality assurance measures, and growing economic pressure, particularly on paediatric radiology, have led to the development of many recommendations and guidelines in many countries and scientific organizations as well as by various health-care providers. The existence of too many different guidelines leads to irritation, puts pressure on organizations and institutions, and renders comparison of scientific and outcomeoriented studies difficult.

Under these circumstances and based on new insights and newly gained knowledge, and incorporating the outcomes of new therapeutic strategies, efforts have been made to unify recommendations and, at the same time, to consider economic needs and to focus on efficacy (without putting at risk true health-care achievements from earlier improvements). In order to facilitate general acceptance throughout the European countries the following discussion was taken up by two European societies, the ESUR (European Society of Uroradiology) and the ESPR (European Society of Paediatric Radiology), in an attempt to create at least a basic consensus statement for the most common and important conditions in paediatric uroradiology. This will allow flexibility and local adaptation without risking quality, and also provide the various institutions with arguments for maintaining a necessary basic level of health care and education in the face of economic pressure.

\section{Procedural recommendations}

The most commonly performed imaging procedures in paediatric uroradiology are US and VCUG.

\section{Ultrasonography}

Over the past decades US has become a high-end diagnostic imaging procedure. Technological improvements including transducers, amplitude coded colour Doppler sonography (aCDS), harmonic imaging, contrast-enhanced US and 3-D US, as well as new approaches (e.g. perineal US), have not only widened the role of US, but have also led to the need for a much more thorough and dedicated education of those who perform US, especially in children. Due to the wide variety of available equipment and users of paediatric US with different levels of education, US in children is performed in very different ways. In order to provide a basic quality level that also enables comparison of data derived from studies at different institutions and in different countries, procedural recommendations were developed indicating the key features of performing US of the paediatric genitourinary tract. These recommendations outline basic requirements and also suggest potential additional techniques and applications, if available and indicated. Technical requirements and patient preparation are also briefly mentioned.

Although many European countries are in the process of establishing a multilevel approach to US applications that defines different requirements both for education and for equipment needs, US, particularly in children, is far from reaching the status of a generally established approach throughout Europe. Therefore these (political) aspects have not been considered in our proposal (Fig. 2).

\section{Contrast-enhanced voiding urosonography (ce-VUS)}

This has been shown to be a safe and reliable imaging tool for detection of VUR. It even shows a higher VUR incidence than conventional VCUG, partly due to the physics of the US microbubbles, and partly due to the longer and unrestricted observation period, as no radiation issues have to be considered. In several countries US contrast media have been approved for paediatric use, and, therefore, in some centres ce-VUS has become a major imaging procedure for VUR assessment. Consensus statements have defined groups where ce-VUS can be considered a valid alternative to conventional VCUG or radionuclide cystography. These are: VUR assessment in screening populations, in girls, and for follow-up. This proposal tries to briefly outline the commonly accepted course of the procedure trying also to include some important technical remarks, such as using saline only from plastic containers (Fig. 3). Further development, particularly of new and more effective contrast media that also 
Fig. 2 Procedural guidelines for standard paediatric urosonography $(C D S$ colour Doppler sonography, (a)CDS amplitudecoded colour Doppler sonography $=$ power Doppler US, ce-VUS contrast-enhanced voiding urosonography, $3 D U S$ three-dimensional US)
Well-hydrated patient, full bladder, adequate equipment \& transducer, training etc

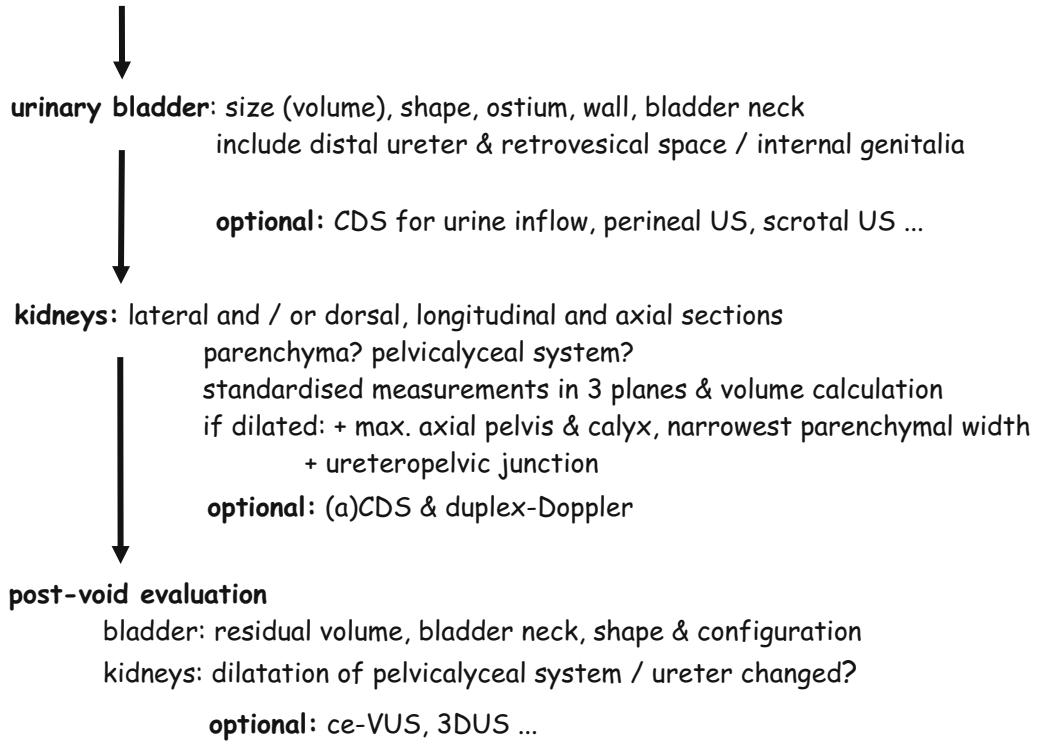

Note: Cursory US of entire abdomen is recommended for first study, and in mismatch of findings and query provide a good acoustic response at higher frequencies, that stay stable for a longer period of time, that are less expensive, and that hopefully will become available for paediatric use in a larger number of countries, will promote the use of this alternative technique that uses no ionizing radiation. A common procedural recommendation may help in establishing this modality. Furthermore, first studies have demonstrated the potential of ce-VUS in the assessment of the urethra.
Voiding cystourethrography

VCUG has been one of the basic investigations in daily paediatric uroradiology for decades. Modern equipment allows pulsed fluoroscopy, fluoroscopic or last-image captures, and digital postprocessing, all of which help to reduce radiation dose significantly for the average procedure. On the other hand, various techniques have been developed, partly driven by time constraints (investigations needed to be
Fig. 3 Procedural guidelines for ce-VUS. (CDS colour Doppler sonography, $H I$ harmonic imaging)

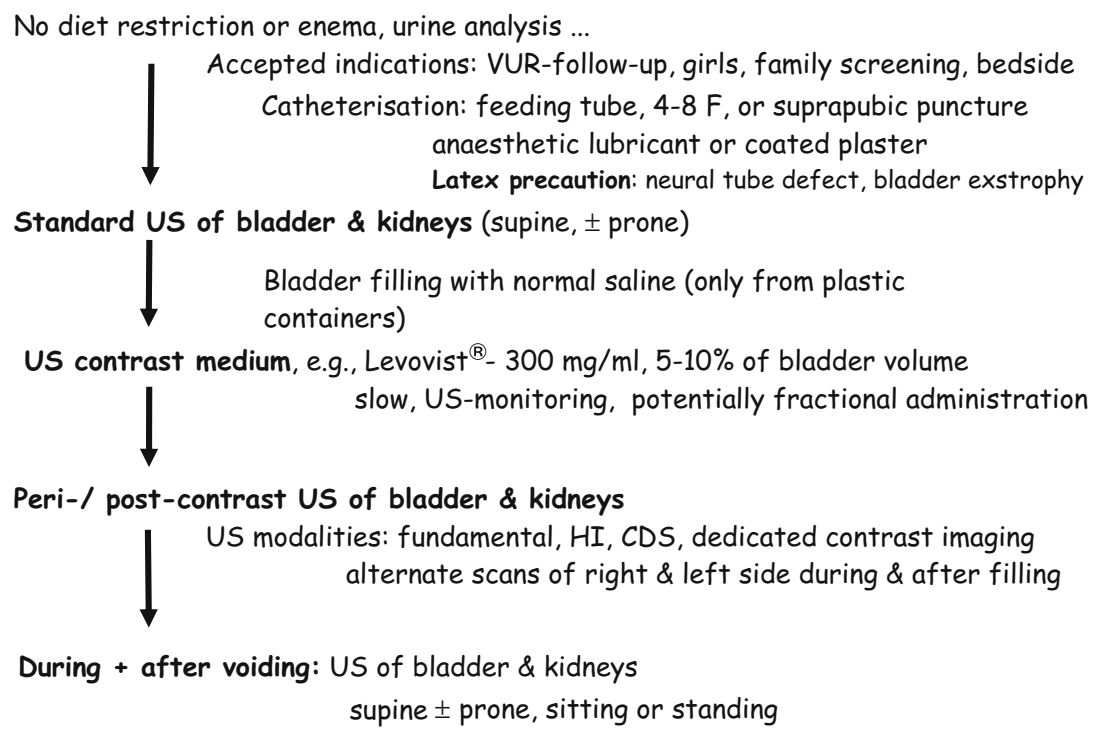

VUR diagnosis: echogenic micro-bubbles in ureters or renal pelves 
Fig. 4 Procedural guidelines for VCUG ( $A B$ antibiotics, $H N$ hydronephrosis, $P U V$ posterior urethral valve, $U T$ urinary tract, UTI urinary tract infection, $V C U G$ voiding cystourethrography, VUR vesicoureteral reflux)
Indications: febrile \& recurrent UTI, particularly in infants, suspected PUV UT-malformation, $\mathrm{HN}>\mathrm{II}^{\circ}$ or "extended criteria"

Preparations: no diet restriction or enema, urine analysis, after $A B$ are completed ... Catheterisation: feeding tube, 4-8 F or suprapubic puncture anaesthetic lubricant or coated plaster Latex precaution: neuro tube defect, bladder exstrophy

Fluoroscopic view of renal fossae \& bladder, initial + early filling

Bladder filling with radio-opaque contrast medium gravity drip; bottle $30-40 \mathrm{~cm}$ above table, watch dripping, $A B$ ?

Fluoroscopy: signs of increased bladder pressure, imminent voiding, urge bilateral oblique views of distal ureters, include catheter document VUR, include kidney (spot film, intra-renal reflux)

When voiding: remove catheter, unless cyclic VCUG $=3$ fillings, $1^{\text {st }}$ y $(s)$ female: 2 spot films of distended urethra (slightly oblique) male: 2-3 spot films during voiding (AP \& steep oblique / lateral) include renal fossae during voiding, if VUR $\Rightarrow$ spot film

After voiding: AP view of bladder \& renal fossae assess contrast drainage from kidney if refluxed

Note: VUR staging, minimise fluoroscopy time and spot films; no control film shorter and shorter), and partly based on new insights (recognition of functional aspects leading to more sophisticated filling techniques and request for post-voiding assessment). Our procedural recommendations for VCUG cover basic patient preparation as well as some remarks on technical requirements. The recommendations illustrate the procedure as a normal flow chart, focusing on the important steps and the relevant minimum number of images required to obtain and document the necessary information and to avoid missing important findings (Fig. 4).

\section{Imaging algorithms}

In the recent past, numerous imaging algorithms have been proposed and developed. This was driven by health-care
Fig. 5 Postnatal imaging algorithm in mild or moderate fetal $\mathrm{HN}$ (IVU intravenous urography, $M C D K$ multicystic dysplastic kidney, $M R U$ magnetic resonance urography)

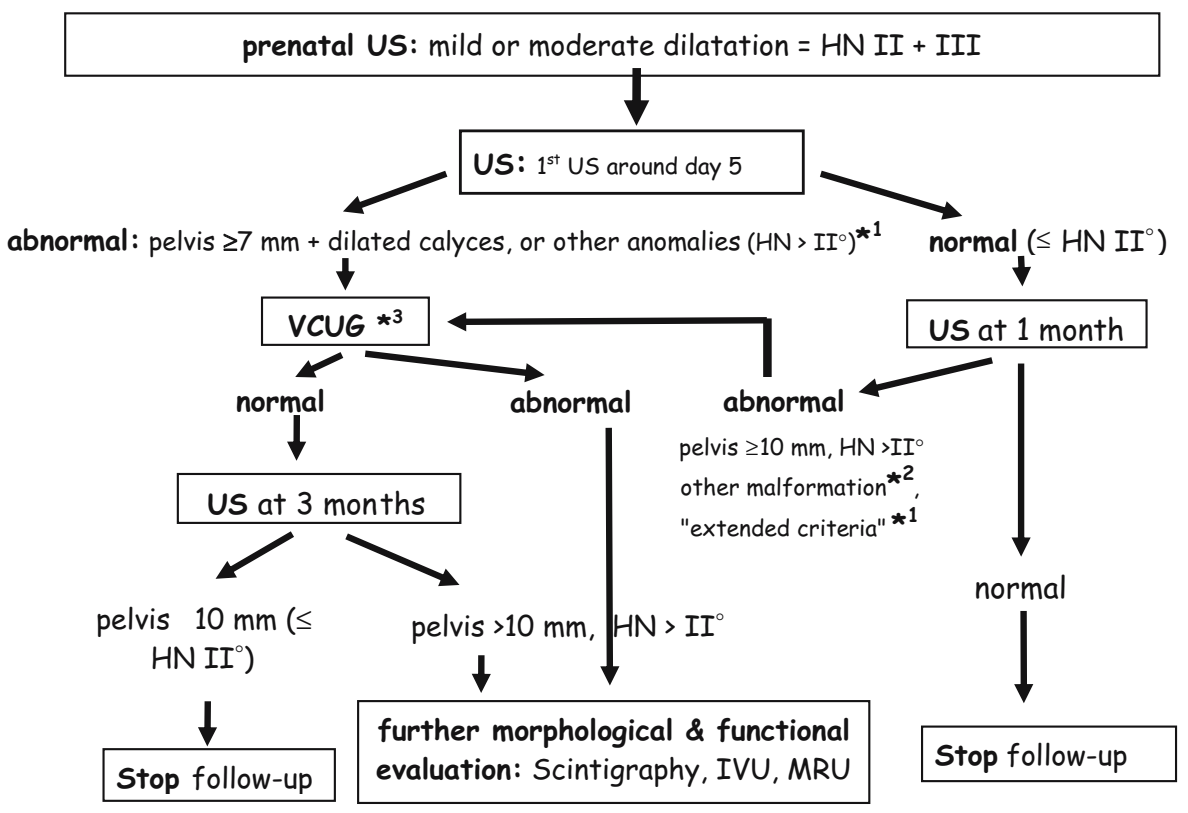

*1 use extended US criteria considering urothelial sign, kidney size \& structure, etc

$\star^{2}$ US genitography: in all patients with single kidney, MCDK, ectopic kidneys etc

${ }^{*}$ ce-VUS can be used in girls and for screening populations 
Fig. 6 Imaging algorithm in children with UTI $(a C D S$ amplitude-coded colour Doppler sonography $=$ power Doppler, $a P N$ acute pyelonephritis, $C R P$ $\mathrm{C}$-reactive protein, $D D$ differential diagnosis, $D M S A$ static renal scintigraphy, $P C N$ percutaneous nephrostomy, $R N C$ radionuclide cystography, $T b$ tuberculosis, $X P N$ xanthogranulomatous pyelonephritis)

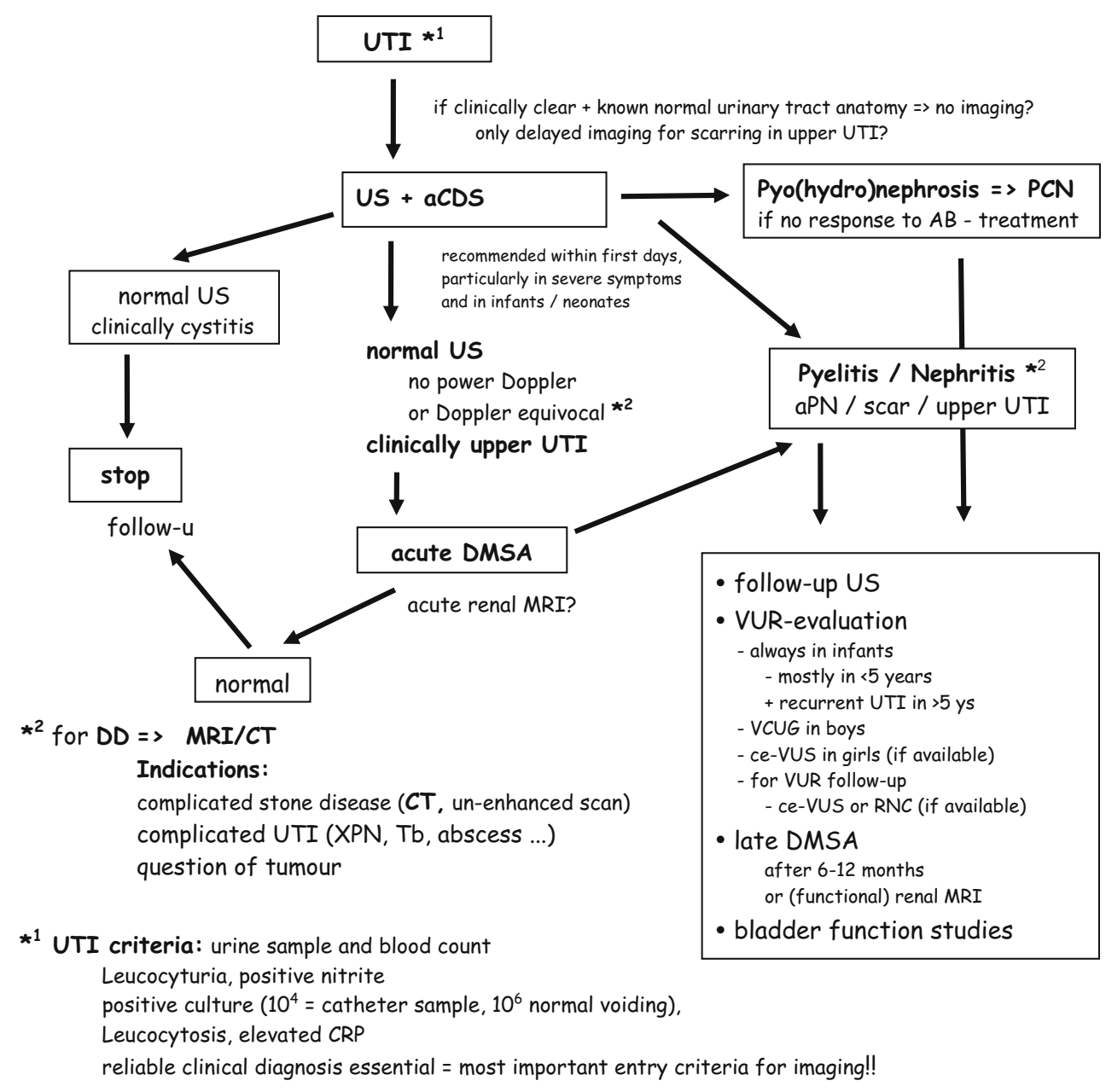

providers and health insurers for medical legal reasons, for reasons of economy, and as a quality measure, but also by various institutions, universities, countries, and scientific societies. An overwhelming number of partially differing imaging guidelines for various clinical settings resulted. With the intention to provide a common European statement that may form the basis for individual and local adaptation as necessary (local availability and expertise, and other needs), the existing imaging algorithms for the two most common conditions in routine paediatric uroradiology (UTI in children, and prenatally detected moderate fetal $\mathrm{HN}$ ) have been reviewed.

With prenatal US as a general screening tool in many countries, a growing number of fetuses in which pelvicalyceal system dilatation has been detected are referred for postnatal assessment. Grading of fetal HN has been standardized by the Society of Fetal Urology (SFU). For intuitive comparison with prenatal findings an adapted HN grading scheme has been developed for postnatal use (Fig. 1). It differs slightly from the prenatal $\mathrm{HN}$ grades by considering aspects that may escape fetal US, particularly forniceal and calyceal configuration. The strategy of postnatal imaging in general follows the final prenatal US. Severe and particularly bilateral fetal urinary tract disease, including (gross) bilateral dilatation, ureteral dilatation, and suspicion of bladder outlet obstruction will initiate an early US investigation and usually a VCUG.

It is more difficult to unify the postnatal workup of mild and moderate fetal HN. Our proposal takes into consideration the fact that the term $\mathrm{HN}$ was developed when prenatal US could only demonstrate pathological dilatation of the pelvicalyceal system. With improved resolution and scanning techniques, physiological filling of the pelvicalyceal system has become visible and is now also described as "low-grade HN". Therefore, as an attempt to avoid unnecessary postnatal imaging in physiological conditions, it is essential not only to reduce the number of unnecessary investigations, but also to avoid invasive procedures and to reduce radiation burden in children. With this in mind, and given the fact that parents often demand postnatal reassurance of an equivocal prenatal US finding, a rather generous indication for US is advised. In order to reduce the number of follow-up studies, US should be performed at 1 week of age, allowing adequate hydration, sufficient renal function 
and urine production, thus preventing underdiagnosis of $\mathrm{HN}$. Based on these findings the further imaging strategy evolves. With equivocal findings, a follow-up US at 46 weeks after birth is advised (Fig. 5).

UTI remains a frequent indication for imaging evaluation of the paediatric urinary tract. The goal of all imaging has been to improve outcome and prevent end-stage renal failure due to scarring from late diagnosis and inadequate treatment. With recognition of the multifactorial genesis of renal scarring, new imaging algorithms focus on renal parenchymal integrity. Only conditions that potentially cause scarring will eventually impair long-term outcome. The role of imaging is to depict and assess children with an increased risk of scarring. Traditionally VUR was seen as the one and only main risk factor for renal scarring in children with UTI; today we know that many other factors are as important.

Based on this new concept, patient management now has several important goals: early and reliable diagnosis of UTI, early and appropriate treatment of diagnosed UTI (different treatment strategies for upper and lower UTI), recognition of complicated disease, detection of bladder function disturbances, and assessment of potentially associated urinary tract abnormality, or VUR. Yet, in spite of prenatal US screening, a number of urinary tract malformations are only diagnosed after symptomatic clinical presentation. Therefore, obviating all imaging in UTI is dangerous. Differentiation of upper and lower UTI may be difficult clinically, and imaging may be requested to confirm or exclude renal involvement. On the other hand, in a child with known normal urinary tract anatomy and a clinically evident diagnosis, early additional imaging may be unnecessary. In given circumstances, initial US is helpful for detection of renal involvement, demonstration of urinary tract anatomy, or early recognition of a complicated course such as abscess formation or pyonephrosis. If the early US (that should include aCDS, if available) answers the relevant questions, treatment (if not initiated yet) must promptly follow in order to avoid renal damage; potential scarring is generally assessed by DMSA scintigraphy after 6 to 9 months or by renal MRI. In children with renal involvement or scar formation, a more thorough investigation, including VUR assessment and bladder function studies, is still indicated. In children without renal involvement or scarring and normal urinary tract anatomy, no additional imaging needs to be performed. There is broad variation in how UTI is diagnosed and how UTI presents clinically, depending on the patient's age and how the clinicians see and deal with the problem. Therefore, some variation of the suggested proposal will persist, affecting the timing and the kind of imaging performed (e.g. availability of scintigraphy or ce-VUS). Our proposal is meant to be a common basis for all further discussions and procedural intentions (Fig. 6). With ever-growing knowledge and new evidence, this proposal will have to be reassessed and updated.

\section{Conclusion and summary}

After presentation of the various items by the individual members of our workgroup, discussions centred on three major aspects:

1. Is there a need to perform postnatal US in babies with prenatal HN I and II at all?

2. Why perform any additional imaging in children with UTI and why not only image those with a complicated course in spite of adequate treatment?

3. How can we establish proper and skilled US at various levels of expertise that naturally should also be reflected in the remuneration system?

Some rather radical proposals differ from the general approach in most European countries. As there is no evidence that omitting existing imaging algorithms does not endanger a number of children and may not diminish long-term outcome, these proposals were generally accepted as a common basic starting point from where we are able to further develop, adapt and update, according to upcoming new knowledge and new evidence, partially enabled by this new common imaging approach. In order to make this evidence available to those who perform imaging evaluation in children, retrospective analysis of our joint experience in many different major institutions and multi-institutional studies will become necessary and should be encouraged. Only if sufficient comparable and relevant data are available for assessing the effects of our imaging approaches on longterm outcome, will we eventually be able to base our future proposals on strict scientific facts and not on a subjective consensus statement.

Other recommendations for further important paediatric conditions are being developed and will be proposed for discussion in the near future, aimed not only at improving quality of patient care, but primarily at enabling the provision of optimal management of all affected infants and children at acceptable cost.

\section{Further reading}

1. Gordon I, Riccabona M (2003) Investigating the newborn kidney update on imaging techniques. Semin Neonatol 8:269-278

2. Riccabona M, Fotter R (2004) Reorientation and future trends in paediatric uroradiology: minutes of a symposium. Pediatr Radiol 34:295-301

3. Riccabona M (2005) Urinary tract infection. In: Carty H, Brunelle F, Stringer D et al (eds) Imaging children, vol 1, 2nd edn. Elsevier Science, pp 691-712

4. Riccabona M, Fotter R (2006) Radiographic studies in children with kidney disorders: what to do and when. In: Hogg R (ed) Kidney disorders in children and adolescents. Taylor and Francis, Birmingham, pp 15-34 
5. Riccabona M, Avni F, Blickman J et al (2006) Guidelines in paediatric uroradiology (abstract). Proceedings of the ESUR Meeting, Cairo, 8-11 September, p 72

6. Darge K (2007) Voiding urosonography with ultrasound contrast agents for the diagnosis of vesicoureteric reflux in children. I. Procedure. Pediatr Radiol. DOI 10.1007/s00247-007-0529-7

7. Avni FE, Hall M, Cassart M et al (2008) The postnatal workup of congenital uronephropathies. In: Fotter R (ed) Pediatric uroradiology, 2nd edn. Springer, Berlin Heidelberg New York, in press

8. Avni FE, Hall M, Cassart M et al (2008) Urinary tract infection. In: Fotter R (ed) Pediatric uroradiology, 2nd edn. Springer, Berlin Heidelberg New York, in press

9. Dacher JN (2008) Diagnostic procedures, excluding MRI, nuclear medicine, and video-urodynamics. In: Fotter R (ed) Pediatric uroradiology, 2nd edn. Springer, Berlin Heidelberg New York, in press 